\title{
Unification of Gravitational and Electromagnetic Fields Using Gauge Symmetry
}

\author{
Young Hwan Yun ${ }^{1 *}$, Kiho Jang ${ }^{1}$, Yong Kiel Sung² \\ ${ }^{1}$ Zero Theoretical Physics Laboratory, Seoul, Republic of Korea \\ ${ }^{2}$ Physical Chemistry Laboratory, College of Science, Dongguk University, Seoul, Republic of Korea \\ Email: *yhyun690@naver.com
}

How to cite this paper: Yun, Y.H., Jang, $\mathrm{K}$. and Sung, Y.K. (2021) Unification of Gravitational and Electromagnetic Fields Using Gauge Symmetry. Journal of High Energy Physics, Gravitation and Cosmology, 7, 344-351.

https://doi.org/10.4236/jhepgc.2021.71018

Received: December 15, 2020

Accepted: January 26, 2021

Published: January 29, 2021

Copyright $\odot 2021$ by author(s) and Scientific Research Publishing Inc. This work is licensed under the Creative Commons Attribution International License (CC BY 4.0).

http://creativecommons.org/licenses/by/4.0/

\section{Open Access}

\begin{abstract}
A new method for the unification of gravitational and electromagnetic forces is proposed. Previously, Kaluza-Klein theory dealt with the unification, but it has not yet been established as a complete theory. The main reason for this is that Kaluza-Klein theory has various contradictions due to the use of a 5-dimensional metric tensor. In this paper, unlike the conventional method, various equations related to gravitational and electromagnetic force are derived without any contradiction by processing equations having gauge symmetry within a 4 -dimensional range. In this process, we propose that Maxwell's equations for the electromagnetic force are expressed more simply and implicitly than the existing tensor form. Using the gauge symmetry, it shows that electromagnetic force can exist in single metric tensor along with gravity. In addition, since geodesic equations can be derived in the form of coordinate transformation, it has been shown that they are consistent with the existing equations. As a result, it has shown that they are consistent with the existing physical equations without contradiction.
\end{abstract}

\section{Keywords}

Gravity, Electromagnetic Field, Kaluza-Klein Theory, Unification, Gauge Symmetry, Relativity

\section{Introduction}

The unification of gravitational and electromagnetic forces is a very interesting study, and there have been attempts to unify gravitational and electromagnetic forces before. In 1914 Finland Nordström discovered that the gravity includes Maxwell's equations in the fifth dimension [1] [2] [3] [4], but he had gotten no attention. In 1921, Kaluza, a mathematician at the University of Konigsberg, 
presented the theory of unifying gravitational and electromagnetic forces [5] by extending the general theory of relativity into five-dimensional space-time. Kaluza was able to further separate the field equations, one of which was equivalent to Einstein's equation, and the other was equivalent to Maxwell's equations for electromagnetic fields. The part of the rest is an additional scalar field called Radion. However, it was not possible to explain how the $5^{\text {th }}$ dimension could exist. But in 1926 Klein explained why the $5^{\text {th }}$ dimension could not be observed by assuming the size reduction [6]. However, there are still many contradictions with the physical equations operated in four dimensions, and in the later superstring theory, ten-dimensional space-time was introduced to resolve these contradictions. However, only four dimensions have been known, and in order to explain the remaining six dimensions, the logic that "the extra spatial dimension is hidden by the size of Planck" has been used in the Kaluza-Klein theory. However, there is still no clear explanation as to why only this extra sixth dimension is so small. In this paper, unlike the previous approaches, we are only trying to solve this problem in a four-dimensional space-time.

\section{Metric Tensor Transformation}

The metric tensor proposed by Kaluza has a $5 \times 5$ structure as follows [6].

$$
g_{\mu \nu}=\left(\begin{array}{ccccc}
g_{00} & g_{01} & g_{02} & g_{03} & k A_{0} \\
g_{10} & g_{11} & g_{12} & g_{13} & k A_{1} \\
g_{20} & g_{21} & g_{22} & g_{23} & k A_{2} \\
g_{30} & g_{31} & g_{32} & g_{33} & k A_{3} \\
k A_{0} & k A_{1} & k A_{2} & k A_{3} & k
\end{array}\right)
$$

This did not satisfy the tensor condition, so Klein supplemented it and proposed it again as follows [6] [7] [8] [9].

$$
g_{\mu v}=\left(\begin{array}{ccccc}
g_{00}+k A_{0} A_{0} & g_{01}+k A_{0} A_{1} & g_{02}+k A_{0} A_{2} & g_{03}+k A_{0} A_{3} & k A_{0} \\
g_{01}+k A_{0} A_{1} & g_{11}+k A_{1} A_{1} & g_{12}+k A_{1} A_{2} & g_{13}+k A_{1} A_{3} & k A_{1} \\
g_{02}+k A_{0} A_{2} & g_{12}+k A_{1} A_{2} & g_{22}+k A_{2} A_{2} & g_{23}+k A_{2} A_{3} & k A_{2} \\
g_{03}+k A_{0} A_{3} & g_{13}+k A_{1} A_{3} & g_{23}+k A_{2} A_{3} & g_{33}+k A_{3} A_{3} & k A_{3} \\
k A_{0} & k A_{1} & k A_{2} & k A_{3} & k
\end{array}\right)
$$

Equation (2-2) can be seen as a more advanced form than Equation (2-1) because it can have the identity as (2-3) [9].

$$
g^{\mu v} g_{\mu \lambda}=\delta_{\lambda}^{v}
$$

However, the $5 \times 5$ tensor has a physical limitation and is an incomplete theory [8] [9] [10]. In contrast, the metric tensor proposed in this paper is of a 4 $\times 4$ format which is different from the above. In the newly proposed tensor, it is denoted as $g_{\mu v}^{\prime}$ as the meaning of the modified metric.

$$
g_{\mu \nu}^{\prime}=\left(\begin{array}{llll}
g_{00}^{\prime} & g_{01}^{\prime} & g_{02}^{\prime} & g_{03}^{\prime} \\
g_{01}^{\prime} & g_{11}^{\prime} & g_{12}^{\prime} & g_{13}^{\prime} \\
g_{02}^{\prime} & g_{12}^{\prime} & g_{22}^{\prime} & g_{23}^{\prime} \\
g_{03}^{\prime} & g_{13}^{\prime} & g_{23}^{\prime} & g_{33}^{\prime}
\end{array}\right)
$$


The inner elements of this metric tensor are as follows.

$$
\begin{gathered}
g_{00}^{\prime}=g_{00}+\varepsilon\left(\partial_{0} \Lambda_{0}+\partial_{0} \Lambda_{0}\right) \\
g_{01}^{\prime}=g_{01}+\varepsilon\left(\partial_{0} \Lambda_{1}+\partial_{1} \Lambda_{0}\right) \\
\vdots \\
g_{33}^{\prime}=g_{33}+\varepsilon\left(\partial_{3} \Lambda_{3}+\partial_{3} \Lambda_{3}\right)
\end{gathered}
$$

In other words, it is a form utilizing Lie derivative [11]. In the Kaluza-Klein theory, they regarded the extended fourth dimension as a type of gauge, but in this theory, it was clearly set as a local gauge. Gauge conversion is defined as follows [12] [13].

$$
x^{i} \rightarrow x^{\prime i}=x^{i}+\varepsilon \Lambda^{i}(x) \text { with } \varepsilon \rightarrow 0
$$

Equation (2-6) transformation expresses a small amount of change in displacement, and this amount of change is expressed as a function of $x$, so it becomes a local gauge. Equation (2-5) is derived from the coordinate transformation process as follows [12].

$$
g^{\prime \mu \nu}=\frac{\partial x^{\prime \mu}}{\partial x^{\alpha}} \frac{\partial x^{\prime \nu}}{\partial x^{\beta}} g^{\alpha \beta}
$$

\section{Check for Symmetry}

As already known, the gravitational equation in general relativity is as the following Equation (3-1). The metric tensor proposed in Equation (2-4) is put into the existing gravitational equation to check the gauge symmetry first [12] [13]. Gauge symmetry is a known fact, but since Maxwell's equations will be derived from this symmetry, so we want to show all derivation directly.

$$
R_{j k}-\frac{1}{2} g_{j k} R=k T_{j k}
$$

where

$$
R_{j k}=\partial_{i} \Gamma^{i}{ }_{j k}-\partial_{j} \Gamma_{i k}^{i}+\Gamma_{i p}^{i} \Gamma_{j k}^{p}-\Gamma^{i}{ }_{j p} \Gamma^{p}{ }_{i k}
$$

where

$$
\Gamma_{j k}^{i}=\frac{1}{2} g^{i l}\left(\partial_{j} g_{k l}+\partial_{k} g_{j l}-\partial_{l} g_{j k}\right)
$$

Here, we will substitute metric tensor Equation (2-5) to Equation (3-3). To find out what the gauge term will be, we can first write Equation (2-5) as one below.

$$
g_{j k}^{\prime}=g_{j k}+\varepsilon\left(\partial_{j} \Lambda_{k}+\partial_{k} \Lambda_{j}\right)
$$

As stated in Equation (2-6), with $\varepsilon \rightarrow 0$, we can get following condition.

$$
g_{j k} \gg\left|\varepsilon\left(\partial_{j} \Lambda_{k}+\partial_{k} \Lambda_{j}\right)\right|
$$

When the gauge term is substituted into the Equation (3-2), the $3^{\text {rd }}$ and $4^{\text {th }}$ terms on the right side have a component of $\varepsilon^{2}$ or more, so the value is even smaller and ignored, and only the $1^{\text {st }}$ and $2^{\text {nd }}$ terms are used. This is the same development as using the linear approximation in the weak gravitational field 
shown in Equation (3-6) [13].

$$
g^{\mu \nu}=\eta^{\mu \nu}+h^{\mu \nu},\left|h^{\mu \nu}\right| \ll 1
$$

In our case, only the $1^{\text {st }}$ and $2^{\text {nd }}$ terms in Equation (3-2) are used, and the gauge term in Equation (3-4) has substituted only in the $1^{\text {st }}$ and $2^{\text {nd }}$ terms in Equation (3-2) with the same logic. The difference between Equation (3-6) and Equation (3-4) when substituted in Equation (3-2) is that in Equation (3-6), $\eta^{\mu v}$ is a constant term, so it disappears from the derivative. On the other hand, $g^{\mu \nu}$ in Equation (3-4) does not disappear. The remaining $O(\varepsilon)$ and $O\left(\varepsilon^{2}\right)$ or more are ignored according to the $\varepsilon \rightarrow 0$ condition stated in Equation (2-6).

Now, to check the gauge symmetry of the $1^{\text {st }}$ and $2^{\text {nd }}$ terms of the Ricci tensor of Equation (3-2), we organize it using Equation (3-3).

$$
\begin{aligned}
& \partial_{i} \Gamma^{i}{ }_{j k}-\partial_{j} \Gamma^{i}{ }^{i} \\
& =\partial_{i} \frac{1}{2} g^{i l}\left(\partial_{j} g_{k l}+\partial_{k} g_{j l}-\partial_{l} g_{j k}\right)-\partial_{j} \frac{1}{2} g^{i l}\left(\partial_{i} g_{k l}+\partial_{k} g_{i l}-\partial_{l} g_{i k}\right)
\end{aligned}
$$

(the gray terms cancel each other out and according to Equation (3-6), we obtain).

$$
\begin{aligned}
& =\partial_{i} \frac{1}{2} g^{i l}\left(\partial_{k} g_{j l}-\partial_{l} g_{j k}\right)-\partial_{j} \frac{1}{2} g^{i l}\left(\partial_{k} g_{i l}-\partial_{l} g_{i k}\right) \\
& \approx \frac{1}{2}\left(\partial^{l}\left(\partial_{k} g_{j l}+\partial_{j} g_{l k}\right)-\partial^{l} \partial_{l} g_{j k}-\partial_{j} \partial_{k} g\right)
\end{aligned}
$$

So, the Ricciscalar, $R=g^{j k} R_{j k}$, is

$$
\begin{aligned}
R & =\frac{1}{2}\left(\partial^{l}\left(\partial^{j} g_{j l}+\partial^{k} g_{l k}\right)-\partial^{l} \partial_{l} g-\partial^{l} \partial_{l} g\right) \\
& =\partial^{l} \partial^{m} g_{l m}-\partial^{l} \partial_{l} g
\end{aligned}
$$

Finally, the Einstein tensor is

$$
\begin{aligned}
& R_{j k}-\frac{1}{2} g_{j k} R \\
& =\frac{1}{2}\left(\partial^{l}\left(\partial_{k} g_{j l}+\partial_{j} g_{l k}\right)-\partial^{l} \partial_{l} g_{j k}-\partial_{j} \partial_{k} g-g_{j k} \partial^{l} \partial^{m} g_{l m}+g_{j k} \partial^{l} \partial_{l} g\right) \\
& =\partial^{l}\left(\partial_{k} g_{j l}+\partial_{j} g_{l k}\right)-\partial^{l} \partial_{l} g_{j k}-\partial_{j} \partial_{k} g-\frac{1}{2}\left(g_{j k} \partial^{l} \partial^{m} g_{l m}-g_{j k} \partial^{l} \partial_{l} g\right)
\end{aligned}
$$

Now, we will check the symmetry of $\varepsilon\left(\partial_{j} \Lambda_{k}+\partial_{k} \Lambda_{j}\right)$ of Equation (3-4). We will do it in contravariant type. Equation (3-11) is the contravariant type of Equation (3-10) [12] [13].

$$
\begin{aligned}
& R^{\mu v}-\frac{1}{2} g^{\mu v} R \\
& =\partial_{\lambda} \partial^{\lambda} g^{\mu \nu}+\partial^{\mu} \partial^{v} g-\partial_{\lambda} \partial^{v} g^{\mu \lambda}-\partial_{\lambda} \partial^{\mu} g^{\nu \lambda}-g^{\mu \nu} \partial_{\lambda} \partial^{\lambda} g+g^{\mu v} \partial_{\lambda} \partial_{\sigma} g^{\lambda \sigma}
\end{aligned}
$$

Substituting $\partial^{\mu} \Lambda^{v}+\partial^{v} \Lambda^{\mu}$ into Equation (3-11),

$$
\begin{aligned}
& R^{\mu v}-\frac{1}{2} g^{\mu v} R \\
& =\partial_{\lambda} \partial^{\lambda}\left(\partial^{\mu} \Lambda^{v}+\partial^{v} \Lambda^{\mu}\right)+\partial^{\mu} \partial^{v}\left(\partial_{\sigma} \Lambda^{\sigma}+\partial_{\sigma} \Lambda^{\sigma}\right)-\partial_{\lambda} \partial^{v}\left(\partial^{\mu} \Lambda^{\lambda}+\partial^{\lambda} \Lambda^{\mu}\right) \\
& -\partial_{\lambda} \partial^{\mu}\left(\partial^{v} \Lambda^{\lambda}+\partial^{\lambda} \Lambda^{v}\right)-g^{\mu v} \partial_{\lambda} \partial^{\lambda}\left(\partial_{\sigma} \Lambda^{\sigma}+\partial_{\sigma} \Lambda^{\sigma}\right)+g^{\mu v} \partial_{\lambda} \partial_{\sigma}\left(\partial^{\lambda} \Lambda^{\sigma}+\partial^{\sigma} \Lambda^{\lambda}\right)
\end{aligned}
$$


Looking at Equation (3-12), there are pairs that cancel each other as follows. In the $1^{\text {st }}$ and $8^{\text {th }}$ terms,

$$
\partial_{\lambda} \partial^{\lambda}\left(\partial^{\mu} \Lambda^{v}\right)-\partial_{\lambda} \partial^{\mu}\left(\partial^{\lambda} \Lambda^{v}\right)=0
$$

In the $2^{\text {nd }}$ and $6^{\text {th }}$ terms,

$$
\partial_{\lambda} \partial^{\lambda}\left(\partial^{v} \Lambda^{\mu}\right)-\partial_{\lambda} \partial^{v}\left(\partial^{\lambda} \Lambda^{\mu}\right)=0
$$

In the $3^{\text {rd }}$ and $4^{\text {th }}, 5^{\text {th }}$ and $7^{\text {th }}$ terms,

$$
\partial^{\mu} \partial^{v}\left(\partial_{\sigma} \Lambda^{\sigma}\right)-\partial_{\lambda} \partial^{\mu}\left(\partial^{v} \Lambda^{\lambda}\right)=0
$$

In the $9^{\text {th }}$ and $11^{\text {th }}$ terms,

$$
-g^{\mu v} \partial_{\lambda} \partial^{\lambda}\left(\partial_{\sigma} \Lambda^{\sigma}\right)+g^{\mu v} \partial_{\lambda} \partial_{\sigma}\left(\partial^{\lambda} \Lambda^{\sigma}\right)=0
$$

In the $10^{\text {th }}$ and $12^{\text {th }}$ terms,

$$
-g^{\mu v} \partial_{\lambda} \partial^{\lambda}\left(\partial_{\sigma} \Lambda^{\sigma}\right)+g^{\mu v} \partial_{\lambda} \partial_{\sigma}\left(\partial^{\sigma} \Lambda^{\lambda}\right)=0
$$

If all of Equations (3-13) to (3-17) are applied, Equation (3-12) becomes as follows.

$$
R^{\mu v}-\frac{1}{2} g^{\mu v} R=0
$$

Therefore, it can be seen that the gravitational equation is symmetric about the gauge transformation expressed as Equation (3-4).

\section{Derivation of Maxwell's Equations}

Applying $g_{\xi_{v}}$ to each of the Equations (3-13) to (3-17) and using the condition $\xi=v$, all converges to the form of the Equation (4-1).

$$
\partial_{\lambda} \partial^{\lambda} \partial_{\sigma} \Lambda^{\sigma}-\partial_{\sigma} \partial^{\sigma} \partial_{\lambda} \Lambda^{\lambda}=0
$$

In short,

$$
\partial_{\lambda} \partial_{\sigma}\left(\partial^{\lambda} \Lambda^{\sigma}-\partial^{\sigma} \Lambda^{\lambda}\right)=0
$$

From now on, we will try to decompose Equation (4-2). The components in parentheses in Equation (4-2) can be defined as one tensor $P^{\lambda \sigma}$ as follows.

$$
P^{\lambda \sigma} \stackrel{\text { def }}{=} \partial^{\lambda} \Lambda^{\sigma}-\partial^{\sigma} \Lambda^{\lambda}
$$

If we apply $\partial_{\lambda}$ on both sides of Equation (4-3),

$$
\partial_{\lambda}\left(\partial^{\lambda} \Lambda^{\sigma}-\partial^{\sigma} \Lambda^{\lambda}\right)=\partial_{\lambda}\left(P^{\lambda \sigma}\right)
$$

If we define the right side of Equation (4-4) as a new tensor $J^{\sigma}$,

$$
J^{\sigma} \stackrel{\text { def }}{=} \partial_{\lambda} P^{\lambda \sigma}
$$

If we take $\partial_{\sigma}$ on both sides of Equation (4-5), according to Equation (4-2),

$$
\partial_{\lambda} \partial_{\sigma} P^{\lambda \sigma}=0
$$

Using Equations (4-5) and (4-6),

$$
\partial_{\sigma} J^{\sigma}=0
$$


In addition, since there is a definition of Equation (4-3), the following identity can be obtained by using it.

$$
\partial^{\alpha} P^{\lambda \sigma}+\partial^{\lambda} P^{\sigma \alpha}+\partial^{\sigma} P^{\alpha \lambda}=0
$$

The above equations are completely similar to the existing electromagnetic equations, which can be summarized as shown in Table 1 [14].

From Table 1, it can be seen that the form of the four equations inferred from the gauge transformation so far is completely consistent with the existing electromagnetic equation, and the basic equation is Equation (4-2). This means Maxwell's Equations can be expressed as one equation as shown in Table 2.

The meaning of equations developed so far is as follows.

When the metric tensor is gauge transformed and applied to the gravitational

Table 1. Relations between electromagnetic equation and gauge transformation equation.

\begin{tabular}{cccc}
\hline & Electromagnetic equations & Gauge equations & \\
\hline Electromagnetic field & $F^{\mu v}=\partial^{\mu} A^{v}-\partial^{v} A^{\mu}$ & $P^{\lambda \sigma}=\partial^{\lambda} \Lambda^{\sigma}-\partial^{\sigma} \Lambda^{\lambda}$ & from (4-3) \\
Field equation & $\partial_{\mu} F^{\mu v}=\frac{4 \pi}{c} j^{v}$ & $\partial_{\lambda} P^{\lambda \sigma}=J^{\sigma}$ & from (4-5) \\
Field equation & $\partial^{\alpha} F^{\mu \nu}+\partial^{\mu} F^{v \alpha}+\partial^{v} F^{\alpha \mu}=0$ & $\partial^{\alpha} P^{\lambda \sigma}+\partial^{\lambda} P^{\sigma \alpha}+\partial^{\sigma} P^{\alpha \lambda}=0$ & from (4-8) \\
Continuous eq. & $\partial_{v} j^{v}=0$ & $\partial_{\sigma} J^{\sigma}=0$ & from (4-7)
\end{tabular}

Table 2. Various types expressing Maxwell's equations.

\begin{tabular}{|c|c|c|c|}
\hline Differential type & Tensor type & Condensed type & \\
\hline \multicolumn{4}{|l|}{$\nabla \cdot D=4 \pi \rho$} \\
\hline$\nabla \times H=\frac{4 \pi}{c} J+\frac{1}{c} \frac{\partial D}{\partial t}$ & $J^{v}=\partial_{\mu} F^{\mu v}$ & & \\
\hline$\nabla \times E=-\frac{1}{c} \frac{\partial B}{\partial t}$ & $0=\partial_{\lambda} F_{\mu \nu}+\partial_{\mu} F_{v \lambda}+\partial_{\nu} F_{\lambda \mu}$ & $\partial_{\lambda} \partial_{\sigma}\left(\partial^{\lambda} \Lambda^{\sigma}-\partial^{\sigma} \Lambda^{\lambda}\right)=0$ & $\begin{array}{l}\text { from } \\
(4-2)\end{array}$ \\
\hline \multicolumn{4}{|l|}{$\nabla \cdot B=0$} \\
\hline$\nabla \cdot J+\frac{\partial \rho}{\partial t}=0$ & $\partial_{\lambda} J^{\lambda}=0$ & & \\
\hline
\end{tabular}

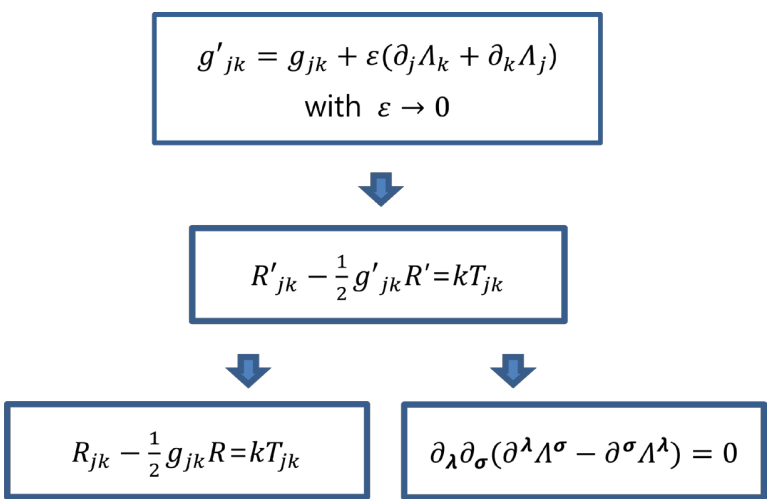

Figure 1. Derivation of Maxwell's equations through Gauge symmetry. 
equations, two equations are obtained: the conventional gravitational equation and the Maxwell's equation as can be seen in the flow of Figure 1. These two equations mean that they are completely independent of each other according to the aforementioned symmetry property, so that they do not have any influence. This is consistent with the physical phenomena so far.

As seen in Equation (2-6), the gauge term is $\Lambda^{i}$, and it can be interpreted as obtaining Maxwell's equations by substituting it with an electromagnetic vector $A^{i}$ of exactly the same form.

\section{Geodesic Equation}

In relativity theory, an important equation along with the gravitational field equation is the geodesic equation. The geodesic equation in the gauge-transformed system (prime coordinate system) is as follows.

$$
\frac{\mathrm{d}^{2} x^{\prime i}}{\mathrm{~d} \tau^{2}}+\Gamma_{j k}^{\prime i} \frac{\mathrm{d} x^{\prime j}}{\mathrm{~d} \tau} \frac{\mathrm{d} x^{\prime k}}{\mathrm{~d} \tau}=0
$$

where [12]

$$
\Gamma_{j k}^{\prime i}=\frac{\partial x^{\prime i}}{\partial x^{v}} \frac{\partial x^{\sigma}}{\partial x^{\prime j}} \frac{\partial x^{\tau}}{\partial x^{\prime k}} \Gamma_{\sigma \tau}^{v}+\frac{\partial x^{\prime i}}{\partial x^{v}} \frac{\partial^{2} x^{v}}{\partial x^{\prime j} \partial x^{\prime k}}
$$

$x^{\prime i}$ in Equation (5-1) follows the definition in Equation (2-6).

The geodesic equation using the Kaluza Equation (2-1) is as follows [9].

$$
\frac{\mathrm{d}^{2} x^{i}}{\mathrm{~d} \tau^{2}}+\Gamma^{i}{ }_{j k} \frac{\mathrm{d} x^{j}}{\mathrm{~d} \tau} \frac{\mathrm{d} x^{k}}{\mathrm{~d} \tau}=-k F^{i}{ }_{j} \frac{\mathrm{d} x^{j}}{\mathrm{~d} \tau} \frac{\mathrm{d} x^{5}}{\mathrm{~d} \tau}-k g^{j 5} A^{i}{ }_{j} \frac{\mathrm{d} x^{j}}{\mathrm{~d} \tau} \frac{\mathrm{d} x^{k}}{\mathrm{~d} \tau}
$$

Also, the geodesic equation using Equation (2-2) supplemented by Klein is as follows [9].

$$
\frac{\mathrm{d}^{2} x^{i}}{\mathrm{~d} \tau^{2}}+\Gamma^{i}{ }_{j k} \frac{\mathrm{d} x^{j}}{\mathrm{~d} \tau} \frac{\mathrm{d} x^{k}}{\mathrm{~d} \tau}=-k F^{i}{ }_{j} \frac{\mathrm{d} x^{j}}{\mathrm{~d} \tau} \frac{\mathrm{d} x^{5}}{\mathrm{~d} \tau}-k A_{k} F^{i}{ }_{j} \frac{\mathrm{d} x^{j}}{\mathrm{~d} \tau} \frac{\mathrm{d} x^{k}}{\mathrm{~d} \tau}
$$

Above both, the metric tensor has 5 dimensions, so the right side is not 0 . In Equation (5-2), the first term on the right side, $k F^{i}{ }_{j} \frac{\mathrm{d} x^{j}}{\mathrm{~d} \tau} \frac{\mathrm{d} x^{5}}{\mathrm{~d} \tau}$ is similar to the Lorentz force term, but the second term $g^{j 5}$ unless it is 0, Equation (2-1) does not become a tensor [9]. Equation (5-3), $\mathrm{kg}^{j 5} \mathrm{~A}_{j}^{i}$ of the $2^{\text {nd }}$ term has no classical correspondence [9].

Since the geodesic equation proposed as a metric tensor in this paper is in the form of coordinate transformation, the problems that appeared in the Kaluza-Klein theory cannot be found as the existing equation form is maintained.

\section{Conclusion}

The four-dimensional metric tensor proposed in this paper was applied to the Einstein tensor to derive both the existing gravitational equation and the Maxwell's equations. Here, a new condensed type of Maxwell's equations has been proposed, which more comprehensively includes a continuity equation other 
than the Maxwell's equations. In addition, when the geodesic equation is used as the proposed metric tensor, it is in the form of coordinate transformation and is applied to the existing equation. From this, we conclude that the metric tensor proposed in this study can overcome the limitations of the metric tensor proposed in the existing Kaluza-Klein theory.

\section{Acknowledgements}

I would like to thank Professor Kook-Hee Kang for introducing several collaborators so that this paper could be submitted.

\section{Conflicts of Interest}

The authors declare no conflicts of interest regarding the publication of this paper.

\section{References}

[1] Isaksson, E. (1985) Gunnar Nordström (1881-1923) on Gravitation and Relativity. XVIIth International Congress of History of Science, 31 July-8 August 1985, Berkeley.

[2] Nordström, G. (1914) Über die Möglichkeit, das lektromagnetische Feld und das Gravitationsfeld zu vereinigen. Physikalische Zeitschrift, 15, 504-506.

[3] Nordström, G. (1914) Zur Elektricitäts- und gravitation-stheorie. Öfversigt af Finska Vetenskaps-Societetens Förhandlingar(Helsingfors), 62A, 1-15.

[4] Nordström, G. (1915) Über eine mögliche Grundlage einer Theorie der Materie. Öfversigt af Finska Vetenskaps-Societetens Förhandlingar (Helsingfors), 62A, 1-21.

[5] Kaluza, T. (1921) Zum Unitätsproblem in der Physik. Sitzungsberichte der Preussischen Akademie der Wissenschaften (Physikalisch-Mathematische Klasse), 1921, 966-972.

[6] Klein, O. (1926) Quantentheorie und fünfdimensionale Relativitätstheorie. Zeitschrift für Physik, 37, 895-906. https://doi.org/10.1007/BF01397481

[7] Duff, M.J. (1995) Kaluza-Klein Theory in Perspective. The Oscar Klein Centenary. Proceedings of the Symposium, 19-21 September 1994, Stockholm.

[8] Van Dongen, J. (1999) Einstein and the Kaluza-Klein particle. Institute for Theoretical Physics, University of Amsterdam Valckeniersstraat 651018 XE Amsterdam and Joseph Henry Laboratories, Princeton University Princeton.

[9] Straub, W.O. (2008) Kaluza Klein Theory. Pasadena, California. http://https//www.physicsforums.com/attachments/kaluza-klein-straub-pdf.55316/

[10] Zaloznik, A. (2012) Kaluza-Klein Theory. University of Ljubljana Faculty of Mathematics and Physics SEMINAR 4. http://mafija.fmf.uni-lj.si/seminar/files/2011 2012/KaluzaKlein theory.pdf

[11] Yano, K. (1957) The Theory of Lie Derivatives and Its Applications. Bibliotheca Mathematica 3, North-Holland.

[12] Ohanian, R. (1994) Gravitation and Spacetime. 2nd Edition, W. W. Norton \& company, New York, 144, 316, 375-376.

[13] Øyvind, G. and Sigbjørn, H. (2007) Einstein's General Theory of Relativity. Springer, New York, 195-214.

[14] John, D.J. (1974) Classical Electrodynamics. University of California, Berkeley, 2. 